\title{
Manejo de la reganancia de peso
}

\author{
Martin E. Rojano-Rodríguez*, Jocabel Chang-Del Ángel y Oscar E. Pérez-Morales \\ Clínica de Cirugía de obesidad, División de Cirugía General y endoscópica, Hospital Dr. Manuel Gea González, Ciudad de México, México
}

\section{Resumen}

Los procedimientos bariátricos se han incrementado en los últimos años. Sin embargo una de las complicaciones a largo plazo es la reganancia de peso que se puede experimentar. La solución de este problema puede conllevar a una reintervención quirúrgica, sin embargo, el riesgo de complicaciones de es mayor incrementando la morbilidad. Razón de ello los métodos endoscópicos pretender solucionar este complicación de manera más segura y eficaz; el objetivos de estos métodos es de restablecer la anatomía quirúrgica reduciendo el tamaño de la anastomosis, o disminuir los reservorios gástricos de la manga gástrica o el pouch gástrico. En la presenta revisión describimos 6 trabajos de poster y uno oral, presentados en la Semana de enfermedades digestivas DDW, de forma digital, en el mes de mayo del año 2020 donde comparan principalmente métodos de reducción de Anastomosis con argón plasma, reducción con sutura endoscópica y con el dispositivo TORe, (Reducción endoscópica transoral de salida). Así como la plicatura con sutura endoscópica Overstich para la reconfección de manga gástrica.

Palabras clave: Endoscopia bariátrica. Reganancia de peso. DDW. Manejo endoscópico de la reganancia de peso.

\section{Introducción}

En diferentes estudios se ha determinando que de 10 hasta un 20 por ciento de los pacientes operados de cirugía bariátrica pueden reganar peso, sin embargo, hay estudios que señalan hasta un $50 \%$ en el caso de manga gástrica ${ }^{1}$.

No existe una definición clara de reganancia, se ha descrito que es la ganancia de 10 kilogramos sobre la pérdida nasal de peso, o bien el recuperar el 25 por ciento de la pérdida del exceso de peso perdido?

Actualmente existen varias técnicas endoscópicas para el tratamiento de reganancia de peso después del bypass gástrico en y de Roux (RYGB). Estos incluyen coagulación con argón plasma (APC) y reducción de la salida transoral (TORe) usando dispositivo de sutura (S-TORe) y un dispositivo de plicatura (P-TORe), los cuales reducen el tamaño de la anastomosis gastroyeyunal (GJA) y/o pouch gástrico. Si bien su eficacia ha sido establecida, no existe un enfoque personalizado para guiar la selección de procedimientos. Así mismo se ha establecido el manejo de la plicatura endoscópica con sutura overstich para realizar nuevamente una manga gástrica sobre la preexistente para tratar la reganancia de peso.

\section{Resultados}

Plicación gástrica endoscópica para el tratamiento de regencia de peso después de bypass gástrico en $Y$ de Roux. (RYGB).

Estudio de prospectivo de pacientes con RYGB que se sometieron a plicatura gástrica (GP) por reganancia de peso. Técnica: Se ablacionó el GJA con plasma de 
argón (APC) (forzado, $0.8 \mathrm{l} / \mathrm{min}, 70-80 \mathrm{~W}$ ). Luego se colocaron plicaturas en el área extirpada y en el pouch. Resultados: Éxito técnico, éxito clínico definido como pérdida de peso total de al menos $5 \%$ (TWL) al año, se informaron eventos adversos (EA). Además, se determinó la cantidad de pérdida de peso, usando\% TWL, a 1 y 2 años.

Resultados: Fueron incluidos 44 pacientes con RYGB (41F, edad 50+_11) se sometieron a GP con reganancia de peso. El promedio de IMC y la reganancia de peso fue $38.8+-7.9 \mathrm{~kg} / \mathrm{m} 2$ y $41.7+-28.6 \%$ de la pérdida de peso máxima. Pre-GP GJA y los tamaños del pouch fueron $17+-7 \mathrm{~mm}$ y $5+-2 \mathrm{~cm}$, respectivamente. Éxito técnico: la tasa de éxito técnico fue del $100 \%$. El tiempo medio del procedimiento fue de 66 a $20 \mathrm{mi}-$ nutos. APC alrededor de la GJA se realizó en todos los pacientes $(100 \%)$ antes de la plicatura $(40.9 \%$ con $80 \mathrm{~W}, 59.1 \%$ con $70 \mathrm{~W}$ ). El número total de plicaturas por caso fue de 6+-3, con 3+-2 y 3+-2 colocados en el GJA y en el pouch respectivamente.

Éxito clínico: la tasa de éxito clínico fue del $79,3 \%$. Al 1 y 2 años, los pacientes experimentaron 10.3+-7.4\% y $8.4+-$ $12.6 \%$ TWL, respectivamente ( $p=0.01$ para ambos).

Seguridad: la tasa general de AE fue de $15.9 \%$. Estos incluyeron estenosis de GJA (13.6\%) y dolor epigástrico debido a la ulceración de GJA en el sitio de APC (2.3\%). Todas las estenosis ocurrieron con 80 W APC y fueron tratados con éxito, mediante dilatación endoscópica con balón o con stent de metal. La tasa de $A E$ severa fue del $0 \%$. Conclusión: El procedimiento de plicatura gástrica parece factible y seguro, con una alta tasa de respuesta y pérdida de peso duradera a los dos años, para el tratamiento de reganancia de peso después de RYGB ${ }^{3}$.

Gastroplastia de manga endoscópica en el tratamiento de la reganancia de peso después de gastrectomía en manga.

Este estudio multicéntrico es el primero en evaluar el uso de la técnica de gastroplastia en manga endoscópica (ESG) para el manejo de reganancia WR después de gastrectomía en manga SG incluyendo pacientes con sobrepeso y obesidad. Pacientes y métodos: Estudio multicéntrico retrospectivo, que incluye pacientes con WR después de SG que se sometieron a ESG para bajar de peso. Los resultados primarios incluyeron AWL, \% TWL, cambio en el IMC, \% EWL a los 6 y 12 meses en pacientes con sobrepeso y obesidad. Éxito clínico se definió en lograr $>25 \%$ de EWL al 1 año con menos del $5 \%$ de eventos adversos graves (SAE) la tasa que sigue el umbral ASGE / ASMBS y $\%$ TWL $>10 \%$. Resultados: 34 pacientes fueron sometidos a ESG después de SG. El éxito técnico fue del 100\%. Al año, $82.35 \%$ y el $100 \%$ de los pacientes alcanzaron> $10 \%$ TWL y $>25 \%$ EWL, respectivamente. En promedio, \%TWL fue $13.2+-3.9$ y $18.3+-5.5$ y \% EWL fue 51.9+19.1 y $69.9+-29.9$ a los 6 meses y al año, respectivamente. \% TWL fue 14.2+-12.5, 19.3+-5.3, 17.5+-5.2, y 20.4+-3.3, y \% EWL fue 88.5+-52.8, 84.4+-22.4, 55.4+14.8 , y $47.8+-11.2$ para sobrepeso, obesidad clase I, II y III, respectivamente a un año de seguimiento. No se identificaron predictores de éxito en el análisis de regresión multivariable. No se reportaron SAE Conclusiones: dado el aumento en el número de gastrectomía en manga realizada en todo el mundo, hay una creciente demanda de una opción de tratamiento endoscópico mínimamente invasivo para los pacientes con reganancia el peso después de SG. Teniendo en cuenta la naturaleza ambulatoria mínimamente invasiva de ESG, la reproducibilidad entre los centros, la baja prevalencia de SAE y los resultados clínicos favorables, ESG después de SG parece ser seguro y efectivo y puede ofrecer una solución para el tratamiento de esta desafiante población de pacientes ${ }^{4}$.

Algoritmo personalizado para la gestión de reganancia de peso después de bypass gástrico en $Y$ de Roux: Sutura vs. coagulación con argón plasma.

Objetivos: 1) evaluar la relación entre las dimensiones anatómicas y el resultado de procedimientos específicos; 2) construir un algoritmo de atención personalizada para el tratamiento de reganancia de peso después de RYGB. Métodos: Diseño del estudio: Análisis retrospectivo de datos recolectados prospectivamente. Nuestro registro hospitalario fue buscando todos aquellos los pacientes que se sometieron a APC, $\mathrm{S}$-TORe y P-TORe. Pacientes que se sometieron revisión endoscópica con disección submucosa o plataformas más antiguas se excluyeron. Análisis: Se realizó un análisis de sensibilidad para determinar la diferencia del (\% TWL) de pérdida de peso total en 12 meses, de cada procedimiento para diferentes tamaños de GJA. Para el pouch $>5 \mathrm{~cm}$, solo se compararon S-TORe y P-TORe, ya que el APC no es típicamente aplicado para reducir el volumen del pouch. Para el pouch $<5 \mathrm{~cm}$, solo APC y S-TORe solo fueron comparados, dado el tamaño del dispositivo de plicatura. ANOVA se utilizó para tres grupos de comparación y la prueba $t$ de Student se utilizó para el análisis de sensibilidad.

Resultados: 751 pacientes cumplieron los criterios de inclusión. De estos, $35.6 \%, 56.1 \%$ y $8.3 \%$ se sometieron a APC, STORe y P-TORe, respectivamente. Las características basales fueron similares entre los grupos. El 
IMC promedio y la reganancia de peso fueron $38.8+-8.4$ $\mathrm{kg} / \mathrm{m} 2$ y $47.4+-37.0 \%$ de la máxima pérdida de peso. Al año, los pacientes de los grupos APC, S-TORe y P-TORe experimentaron $5.1+-9.4 \%, 7.6+-8.4 \%$ y $8.2+-$ $7.5 \%$ TWL, respectivamente $(p=0.001)$. Parte I: Pouch $>5 \mathrm{~cm}$ (S-TORe vs. P-TORe). En el análisis de sensibilidad, con un tamaño de GJA de $30 \mathrm{~mm}$, S-TORe resultó en una mayor pérdida de peso que P-TORe (11.9+-7.6\% vs. $2.7+-1.4 \%, p=0.03$ ). Sin embargo, con un tamaño de GJA $<30 \mathrm{~mm}$, P-TORe presento mayor pérdida de peso que S-TORe (11.1+-8.5\% vs. $7.9+-7.5 \%$ TWL, $p=0.05$ ). Parte II: Pouch $\leq 5 \mathrm{~cm}$ (APC vs. S-TORe). En el análisis de sensibilidad, con un tamaño de GJA de $18 \mathrm{~mm}$, S-TORe presentó una mayor pérdida de peso que APC $(7.6+-8.1 \%$ vs. $5.8+-9.0 \%$ TWL, $p=$ 0.05). Sin embargo, con un tamaño GJA $<18 \mathrm{~mm}$, no hubo diferencia en\% TWL entre APC y S-TORe (5.2+$9.1 \%$ vs. $5.7+-10.2 \%$ TWL, $p=0.77$ ).

Parte III: Basado en las partes I y II, se construyó un algoritmo Conclusión: El tratamiento endoscópico de reganancia de peso después de RYGB se puede individualizar según la anatomía de los pacientes, para un pouch $>5 \mathrm{~cm}$, se debe considerar P-TORe cuando GJA es $<30 \mathrm{~mm}$, con S-TORe siendo realizado cuando GJA es de $30 \mathrm{~mm}$. Para el pouch $<5 \mathrm{~cm}$, se pueden considerar tanto APC como S-TORe para GJA $<18 \mathrm{~mm}$, prefiriéndose S-TORe cuando GJA es $18 \mathrm{~mm}^{5}$.

Resultados de siete años de reducción de la salida transoral (TORe) para el tratamiento de la reganancia de peso después de bypass gástrico en y de Roux.

Diseño del estudio: Revisión retrospectiva de datos recolectados prospectivamente sobre pacientes con RYGB que se sometieron a TORe por reganancia de peso.

Técnica: TORe se realizó utilizando un dispositivo de sutura endoscópica de espesor completo. La GJA se ablacionó usando coagulación con plasma de argón. Se colocaron puntos alrededor del GJA para reducir su tamaño. Resultado primario: Porcentaje de pérdida de peso total (\% TWL) a los 7 años después de TORe. Resultados secundarios: detalles del procedimiento, perfil de seguridad clasificado con el léxico ASGE, número necesario para tratar (NNT) para detener la recuperación de peso y predictores de\% TWL a los 7 años después de TORe. Resultados: Un total de 443 pacientes con RYGB se sometieron a TORe y fueron incluidos en el estudio. La edad basal, el peso y el IMC fueron $49 \pm 11$ años, $116.9 \pm 28.4 \mathrm{~kg}$ y $41.8 \pm 10.5 \mathrm{~kg} / \mathrm{m}^{2}$. En promedio, los pacientes tenían $7 \pm 3$ años post-RYGB y habían recuperado $43.9 \pm 26.3 \%$ de la pérdida máxima de peso. El tamaño de GJA previo a TORe fue de $25.0 \pm 6.4 \mathrm{~mm}$, que disminuyó a $7.2 \pm 2.6 \mathrm{~mm}$ después de TORe. Resultados primarios: Los pacientes perdieron $9.5 \pm 9.5 \mathrm{~kg}, 8.6 \pm 11.7,10.5 \pm 17.5 \mathrm{~kg}$ y 10.1 $\pm 16.0 \mathrm{~kg}$ a $1,3,5$ y 7 años, respectivamente $(p<0.01$ para todos). Esto correspondió a $7.5 \pm 6.6 \%, 6.9 \pm$ $8.9 \%, 7.5 \pm 14.4 \%$ y $8.4 \pm 12.8 \%$ TWL a $1,3,5$ y 7 años con tasas de seguimiento del $86 \%, 94 \%, 86 \%$ y $72 \%$ de pacientes elegibles para el seguimiento en estos puntos de tiempo, respectivamente. Resultados secundarios: el $80.3 \%$, el $16.1 \%$ y el $3.6 \%$ de los casos se realizaron con patrones de sutura de cuerda simple, interrumpida y doble respectivamente, con un promedio de $10 \pm 4$ puntos (bites) por GJA. La sutura de refuerzo del pouch se realizó en $62 \%$, con un promedio de $3 \pm 2$ puntos por pouch. No hubo eventos adversos graves. A los 7 años, el $32 \%$ de los pacientes recibió una terapia de pérdida de peso adicional que incluyó farmacoterapia (18\%), repetición de TORe $(10 \%)$ y revisión quirúrgica (4\%). El NNT para detener la recuperación de peso a los 7 años fue de 1.3. La cantidad de pérdida de peso a 1 año $(\beta=0.67 ; p=0.03)$ fue un predictor de\% TWL a los 7 años después de controlar la edad, el sexo, la recuperación de peso y el uso de terapia complementaria. Conclusiones: TORe parece seguro, efectivo y duradero para tratar el aumento de peso después de RYGB, con un NNT de 1.3. La cantidad de pérdida de peso al año predice la eficacia a los 7 años y puede ayudar al uso directo de la terapia adyuvante $^{6}$.

Manga en manga endoscópica versus manga quirúrgica en pacientes con recuperación de peso después de la gastrectomía en manga: un análisis comparativo retrospectivo.

Estudio retrospectivo de centro único de pacientes adultos con antecedentes de reganancia de peso después de una gastrectomía en manga que se sometieron a SIS (2018-2019) y SRS (2015-2019). EI SIS se realizó con un novedoso sistema operativo sin incisiones que permite la creación de múltiples plicaturas de tejido que reducen el tamaño de la manga. Los datos basales se recolectaron por edad, género, Perdida total de peso corporal (\% PTP) después de MG y peso antes de la revisión. Los resultados primarios fueron \% PTP a los 1, 3, 6 y 12 meses posteriores al procedimiento. Los resultados secundarios incluyeron la duración de la estancia hospitalaria y la tasa de eventos adversos relacionados con el procedimiento. Los análisis estadísticos se realizaron mediante la prueba $T$ de Student o la prueba exacta de Fisher con significación estadística definida como $p$ $<0.05$. Resultados: se incluyeron un total de 18 
pacientes en este análisis (9 pacientes con SIS y 9 pacientes con SRS). Los datos demográficos basales se resumen en la tabla 1. La edad media fue de $45 \pm 11$ años, el $73 \%$ eran mujeres. No hubo diferencias basales en la edad y el sexo, sin embargo, los pacientes que se sometieron a SRS tuvieron pesos de gastrectomía antes de la manga más altos (317 \pm 48 frente a $262 \pm 45 ; p=0.02$ ) y un peso medio más alto antes de la revisión ( $266 \pm 35$ frente a $217 \pm 33$; $p=0.007$ ). El \% de PTP a los 1, 3, 6 y 12 meses posteriores al procedimiento fue similar entre SIS y SRS ( $p>0.05$ ), Tabla 2. Los pacientes con SIS tuvieron una estancia hospitalaria más corta $(1.1 \pm 0.3$ días versus $2.38 \pm 1.41$ días; $p=0.02$ ) y presentaron eventos adversos relacionados a los procedimientos de forma similar $(11.1 \%$ vs. $44.4 \% ; p=0.11)$. Conclusiones: los resultados endoscópicos de manga en manga tienen una eficacia similar a corto plazo en comparación con la nueva manga quirúrgica, con una estadía hospitalaria más corta. Se observó una tasa de eventos adversos más baja, pero no alcanzó significancia estadística, posiblemente debido a que este es un estudio de baja potencia?

Uso del dispositivo Overstitch para la revisión endoscópica del bypass gástrico en $Y$ de Roux: una actualización de la serie más grande del Reino Unido.

Los pacientes fueron discutidos en una reunión bariátrica multidisciplinaria que ya se habían sometido a una gastroscopia diagnóstica para evaluar la idoneidad para el procedimiento. Los criterios de inclusión endoscópica fueron un estoma de al menos $2 \mathrm{~cm}$ de diámetro y una longitud de la bolsa mayor de $3 \mathrm{~cm}$. Cuarenta pacientes (38 mujeres) con BGYR previo y reganancia de peso que cumplían con los criterios endoscópicos se incluyeron con un tamaño de estoma media de $4 \mathrm{~cm}$. Inicialmente, la mucosa de la salida se ablacionó con coagulación con plasma argón (CPA) seguido por el uso del dispositivo overstitch. El patrón de sutura elegido fue mixto con una media de 2 suturas por paciente y un diámetro de estoma medio final de $2.9 \mathrm{~mm}$. Todo el procedimiento se realizó bajo anestesia general con el paciente en posición supina. Después del procedimiento, los pacientes siguieron una dieta estricta bajo supervisión y también se prescribió un tratamiento sintomático preventivo. Resultados: todos los pacientes se sometieron al procedimiento de forma segura sin mayores complicaciones. Solo dos pacientes presentaron dolor abdominal y náuseas que se controlaron fácilmente con medicamentos antieméticos y analgésicos. Solo uno se sometió a la técnica dos veces debido a suturas sueltas. Después de 1 año de seguimiento, se observó una pérdida de peso promedio de $8.9 \mathrm{~kg}$. Conclusión: la revisión endoscópica del BGYR utilizando el dispositivo overstitch es un método efectivo y seguro para lograr una mayor pérdida de peso en pacientes que han recuperado peso después de la cirugía y tienen un estoma que cumple con los criterios endoscópicos. El efecto de pérdida de peso se mantuvo a 1 año después del procedimiento. Es necesario determinar los beneficios a más largo plazo, pero es posible repetir el procedimiento en aquellos pacientes que recuperan peso ${ }^{8}$.

Repetir la reducción de la salida transoral (desgarro) para pacientes con recuperación de peso después del bypass gástrico Roux-en-y (BGYR)

Métodos: Esta es una revisión retrospectiva de datos recolectados prospectivamente, para todos los pacientes que se sometieron a TORe con overstitch Apollo de 2010 a 2018 en nuestro centro.

Resultados: Se identificaron 321 pacientes que tenían al menos un procedimiento TORe. Entre ellos, 23 pacientes $(7 \%)$ recibieron TORe repetidos y se incluyeron en el análisis. La edad promedio fue $49( \pm 10)$ años, $83 \%$ mujeres y un tiempo total de seguimiento de 5 ( \pm 2) años. Se consideraron dos grupos de pacientes para TORe repetidos. El primero, los no respondedores, definidos como aquellos que no pudieron alcanzar el $5 \%$ de PTP a los 12 meses después del primer TORe (11 pacientes). El segundo grupo son los respondedores, que reganaron peso después de una pérdida de peso adecuada inicial ( $\geq 5 \%$ ) (12 pacientes). En un año después del índice TORe, los pacientes que fueron clasificados como respondedores perdieron un promedio de - $28 \mathrm{lbs}( \pm 14 \mathrm{lbs}$, PTP de $-11 \%, p<0.001)$. En comparación con aquellos que fueron clasificados como no respondedores que perdieron $-4 \mathrm{lbs}( \pm 7 \mathrm{lbs}$, PTP de $+3 \% ; p=0.14)$. Ambos grupos se sometieron a repetición de TORe (dentro de un promedio de 3.5 años y 2.3 años para respondedores y no respondedores respectivamente del índice TORe). Un año después de la repetición de TORe, los respondedores perdieron con éxito un promedio de $-27 \mathrm{lbs}( \pm 21 \mathrm{lbs}$, PTP de $-11 \%$, $p<0.001)$. En comparación con los no respondedores que reganaron un promedio de $5 \mathrm{lbs}( \pm 14 \mathrm{lbs}$, PTP de $-3 \% ; p=0.32$ ). Ambos grupos fueron seguidos durante un promedio de 2-3 años después de la repetición de TORe. Los respondedores habían sostenido una PTP de $-10 \%(p=0.002)$ mientras que los no respondedores tenían una PTP de -4\% (0.13) en comparación con el peso antes del procedimiento repetido de, TORe. Los no respondedores tenían un IMC promedio más bajo antes al índice TORe (34 versus $41 ; p=0.02$ ) y es más probable que haya tenido un patrón de sutura 
interrumpido durante el índice TORe (45\% versus $17 \%$; $p=0.13)$, Tabla 1 .

Conclusión: la repetición del procedimiento TORe es una opción razonable para aquellos que recuperaron peso después de una respuesta inicial adecuada al índice TORe. Por otro lado, aquellos que no respondieron al índice TORe deberían considerarse para opciones alternativas como medicamentos contra la obesidad o revisión quirúrgica ${ }^{9}$.

\section{Conclusiones}

Existen varios métodos endoscopios para tratar la reganancia de peso. El objetivo principal de estos métodos es restablecer la anatomía quirúrgica inicial para inducir el efecto en la pérdida de peso. Los métodos principalmente de sutura muestran resultados eficaces con seguridad, colocándose en la primera línea en el manejo de la reganancia de peso antes del los procedimientos quirúrgicos, ya que a la vez presentan menor morbilidad.

\section{Financiamiento}

Los autores no recibieron patrocinio para llevar a cabo este artículo.

\section{Conflicto de intereses}

Los autores declaran no tener conflicto de intereses alguno.

\section{Bibliografía}

1. Tran DD, Nwokeabia ID., Purrell S, Zafar SN, Ortega G, Hughes K, Fullum TM. Revision of Roux-En-Y Gastric Bypass for Weight Regain: a Systematic Review of Techniques and Outcome. Obesity Surgery 2016:26;1627-1634.

2. Luti M, Kularatna M, Hill AG, MacCormick AD. Weight Regain Following Sleve Gastrectomy- a Systematic Review. Obesity Surgery 2016: 26;1326-1334.

3. Jirapinyo P, Thompson ChC, Gastrointestinal endoscopy2020:91(5S);AB212

4. Moura DT, Barrichello S, Moura EG, Souza TF, Galvao M, Grecco E, et al. Endoscopic Sleeve Gastroplasty in the management of Weight Regain after sleeve Gastrtectomy. Gastrointestinal endoscopy 2020:91(5S);AB210.

5. Jirapinyo P, Thompson Ch.C, Personalizer Algorithm for The Management of Weight regain following Roux-en-Y Gastric bypass: Suturing versus plication Argon plasma coagulation. Gastrointestinal endoscopy 2020: 91(5S);AB10.

6. Jiraoinyo P, Maahs ED, Thompson Ch. C. Seven Year outcomes of transoral Outlet Reduction (TORE) For the Treatment of Weight regain following roux-en-Y Gastric Bypass Gastrointestinal endoscopy 2020: 91(5S);AB212.

7. Najdat Bazarbashi A., Jirapinyo P., Thompson Ch. C., Endoscopic Sleeve-in-Sleeve versus surgical Re-Sleeve in patients with weight regain after Sleeve Gastrectomomy: A Retrospective Comparative Analysis. Gastrointestinal endoscopy 2020:91(5S);AB226.

8. Prades L.F., Ahmed A., Lopes M.M, Kaur V, Bansi D, Use of the Overstich device for endoscopic revision of Roux-En-Y Gastric Bypass: An Update from the Largest UK Series. Gastrointestinal endoscopy 2020: 91(5S);AB231.

9. Abbas AM, Jirapinyo $P$, Hathorn $K$, MacCarty TR, Thompson Ch C. Repeat transoral outlet Reduction (TORE) For Patients with weight Regain Following Roux-en-Y Gastric Bypass (RYGB). Gastrointestinal endoscopy 2020:91(5S);AB216. 